\title{
Energy Dispersive X-Ray Spectroscopy in Liquids: Inorganic and Biological Applications
}

\author{
D. J. Kelly ${ }^{1}$, E. A. Lewis ${ }^{1}$, H. Downie ${ }^{2}$, J. R. Lloyd ${ }^{2}$, N. J. Zaluzec ${ }^{1,3}$, M. G. Burke ${ }^{1}$ and S. J. Haigh ${ }^{1}$ \\ ${ }^{1}$ School of Materials, The University of Manchester, Manchester, M13 9PL, United Kingdom. \\ ${ }^{2}$ School of Earth, Atmospheric and Environmental Sciences, University of Manchester, United \\ Kingdom. \\ ${ }^{3}$ Electron Microscopy Centre, Argonne National Laboratory, USA.
}

Combining energy dispersive X-ray spectroscopy (EDS) with liquid-cell scanning transmission microscopy (STEM) enables us to examine the structure and chemical composition of nanostructures in liquids. Liquid-phase spectrum imaging can be used to map elemental distribution and observe chemical changes in multi-metallic systems at the nanoscale.

Silicon-nitride windowed liquid-cells can be used for high resolution imaging of nanostructures in liquids, however EDS analysis is limited by the geometry of the cell set up. We have demonstrated the use of a modified liquid-cell which decreases X-ray shadowing, making nanometer resolution elemental mapping possible in liquids for the first time (Fig. 1) [1].

Studies of complex mixtures of inorganic nanostructures have demonstrated the technique's ability to simultaneously map multiple elements and observe process such as the growth of core-shell nanostructures, providing insights into the complex growth mechanisms associated with the formation and evolution of nanostructures in liquid [2]. Liquid cell EDS can also be applied to the study of hydrated biological specimens [3]. Bacterial reduction of metal salts is of interest as a low cost and environmentally benign synthetic route to nanomaterials. We have studied AuPd nanoparticle decorated bacterial cells synthesised by the co-reduction of $\mathrm{Pd}(\mathrm{II})$ and $\mathrm{Au}(\mathrm{III})$ by Geobacter sulfurreducens cells. Knowledge of the distribution of elements, on both the cellular and individual nanocrystal scale, could give considerable insights into both the mechanism of biological nanocrystal synthesis and the structureproperty relationships in bio-nanoparticle catalysts. We combine in situ experiments on hydrated specimens in the liquid-cell with experiments in vacuum, demonstrating the complementary nature of these two approached [4]. 
References:

[1] NJ Zaluzec et al, Microsc Microanal 20 (2014), pp. 323-329.

[2] EA Lewis et al, Chem Commun 50 (2014), pp. 10019-10022.

[3] EA Lewis et al, Part Part Syst Charact (2016), pp. 1-9.

[4] The authors acknowledge funding from the Engineering and Physical Sciences Research Council (EPSRC) and Defence Threat Reduction Agency.
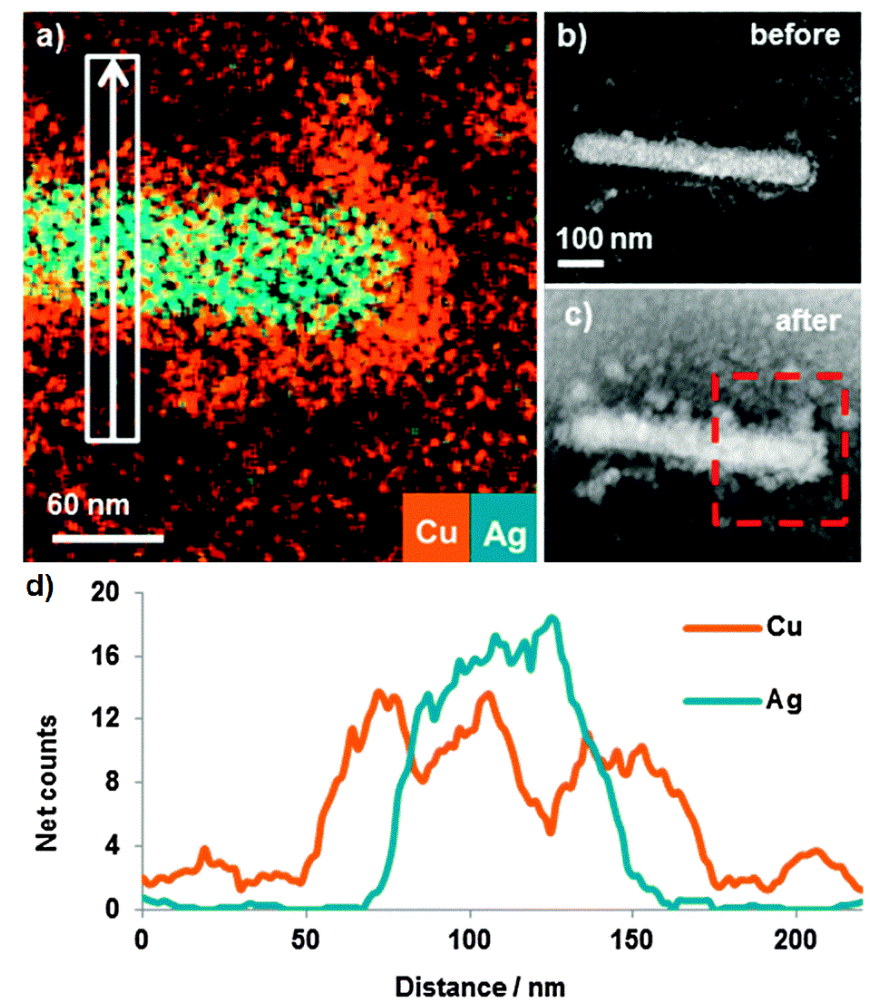

Figure 1. A bimetallic nanostructure in water was imaged before (b) and after (c) XEDS data acquisition, nanoparticle deposition has clearly occurred on and around the Ag-NW during data acquisition. Elemental map (a) extracted from the XEDS spectrum image, for the region of interest indicated by the dotted line in (c), shows an Ag-NW coated in $\mathrm{Cu}$ nanoparticles. An X-ray line profile (d) extracted from the spectrum image shows elevated $\mathrm{Cu}$ concentration on and around the Ag-NW [2], with permission from The Royal Society of Chemistry. 